\title{
Recurrence of Intravenous Leiomyomatosis: A Rare Cause of the Obstruction of the Right Heart
}

Recurrence of Intravenous Leiomyomatosis

\author{
Yopie Afriandi Habibie and Dudy Hanafy
}

Division of Thoracic, Cardiac \& Vascular Surgery, Department of Surgery, Faculty of Medicine, Syiah Kuala University, Dr. Zainoel Abidin General Hospital, Banda Aceh, Indonesia

Division of Adult Cardiac Surgery, Department of Thoracic, Cardiac \& Vascular Surgery, Faculty of Medicine, Indonesia University, National Cardiovascular Center Harapan Kita, Jakarta, Indonesia

\begin{abstract}
Purpose - Intravenous leiomyomatosis (IVL) is a very rare subtype of leiomyoma, involving the right obstruction of the heart, and is an unusual cause of outflow tract obstruction. The IVL grows from the vessel's smooth muscle, protruding into the vessel's lumen, and can expand to the right atrium and even beyond causing death due to blood flow obstruction into the right atrium and even to pulmonary artery.

Design/Methodology/Approach - We present a 33-year-old Indonesian woman with cardiopulmonary symptoms predominantly, and marked by an intravascular leiomyoma extending from inferior vena cava (IVC) to right chamber atrium, with the chief complaint being easily fatigued since seven months.

Findings - Echocardiography observations found a mass in the right atrium and the IVC that caused dynamics obstructed in tricuspid valve, right atrium and ventricle were dilated, no left ventricular (LV) hypertrophy, normal LV and right ventricular (RV) functions, and no valve abnormality except the tricuspid valve gradient being $21 \mathrm{mmHg}$ with mild regurgitation. Venography resulted in a mobile tumor mass in IVC mouth which partially flew into the right atrium, and partially blocked the IVC mouth. Tumor size was $6.4 \mathrm{~cm} \times 4.8 \mathrm{~cm}$. Abdominal multislice computed tomography resulted in a residual soft tissue mass (leiomyoma) along the IVC extended to the right atrium. The tumor mass size in the IVC and the right atrium was bigger compared to tumor mass on July 2008. The correct diagnosis was established during surgery; therefore a two-stage resection was done.

Originality/Value - Surgical resection is the best treatment for intracardiac extension of intravenous leiomyoma. To remove the ilio-caval portion, iliac venotomy was recommended for the tumor in both stages of the surgeries.
\end{abstract}

Keywords Recurrence of intravenous leiomyomatosis, Right heart outflow obstruction

All papers within this proceedings volume have been peer reviewed by the scientific committee of the Malikussaleh International Conference on Multidisciplinary Studies (MICoMS 2017).

(C) Yopie Afriandi Habibie, Dudy Hanafy. Published in the Emerald Reach Proceedings Series. Published by Emerald Publishing Limited. This article is published under the Creative Commons Attribution (CC BY 4.0) licence. Anyone may reproduce, distribute, translate and create derivative works of this article (for both commercial and non-commercial purposes), subject to full attribution to the original publication and authors. The full terms of this licence may be seen at http:// creativecommons.org/licences/by/4.0/legalcode

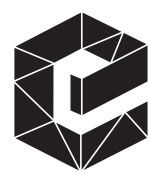

Emerald Reach Proceedings Series Vol. 1 Emerald Publishing Limited 2516-2853 DOI 10.1108/978-1-78756-793-1-00004 


\section{Introduction}

Intravenous leiomyomatosis (IVL) is a very rare subtype of leiomyoma, involving the right heart, and is an unusual cause of outflow obstruction. Evaluation of the patient should not be directed only at intracardiac chambers but also at the inferior vena cava (IVC). This should include angiography, magnetic resonance imaging (MRI), or computed tomography (CT) scanning. Echocardiography is an important contributory investigation. Surgical therapy should be directed at complete removal of the tumor, as recurrence will be reported if a less than complete resection has been performed. Hormonal manipulation has been attempted with variable results (Rosenberg et al., 1988; Galvin et al., 2010).

This tumor in some cases, also known as benign metastasizing leiomyoma, is characterized by uterine leiomyomas associated with the development of the same histological tumor in other organs, such as lung, lymph nodes, central nervous systems, bones, mesentery, deep soft tissues, and heart (Maylar et al., 2009; Galvin et al., 2010). The IVL most frequently affects women between the ages of 40 and 60 years. Most of the tumors arise from the uterus, the large veins (IVC and pelvic veins), and the lungs. The IVL is also a quite expansive tumor. This tumor is a sex hormone-dependent tumor and tumor tissues themselves are a source of estrogen that can support their own growth, without considering ovarian-produced estrogen (Rosenberg et al., 1988; Maylar et al., 2009).

Right heart obstruction of blood flow in the heart is an uncommon entity. Most cases are secondary to rheumatic heart disease with the involvement of the mitral and tricuspid valves. A second, far less common etiology is tumor. The IVL grows from vessel's smooth muscle, protruding into the vessel's lumen, and can expand to right atrium and even beyond causing death due to blood flow obstruction into right atrium and even to pulmonary artery (Filsoufi et al., 2002). While most cardiac tumors are myxomas and left sided, we report a case of RV inflow obstruction secondary to intracardiac IVL ( Rosenberg et al., 1988; Filsoufi et al., 2002; Maylar et al., 2009; Galvin et al., 2010). We present a 33-year-old Indonesian woman with cardiopulmonary symptoms predominantly, and marked by an intravascular leiomyoma extending from the IVC to the right chamber atrium.

\section{Clinical summary}

A 33-year-old exhausted Indonesian woman was admitted to the emergency room due to syncope and shortness of breath since four days. She also had dyspnoea since three months before admission. No chest pain, palpitation, hypertension, diabetes mellitus, or dyslipidaemias was reported. She had a history of taking hormonal chemicals as contraception agents since 2003 because of primary infertility. She complained about easy fatigueness since seven months. The patient was our old patient.

In 2008, she already had intravascular leiomyoma extirpation. She had also undergone uterine leiomyoma extirpation in the same year. She was admitted to our institution following similar symptoms like syncope. Thorax CT-scan was done, which initially diagnosed right atrial myxoma. Abdominal CT-scan was not done. After her general condition was improved, operation was performed, and we obtained a $35-\mathrm{cm}$ tumor in the right atrium, right ventricle until the right pulmonary artery. Although the tumor was completely excised, there still remained a 10-cm residue in the IVC, and histopathology revealed a vascular leiomyoma. She was discharged two weeks postoperation.

In latter admission, she was admitted to in-patient, then chest X-ray, venography, abdominal multislice computed tomography (MSCT), echocardiography, and transesophageal echocardiography (TEE) were done (Figure 1).

The echocardiography observations found a mass in the right atrium and the IVC, right atrium and ventricle were dilated, no LV hypertrophy, normal LV and RV functions, no 


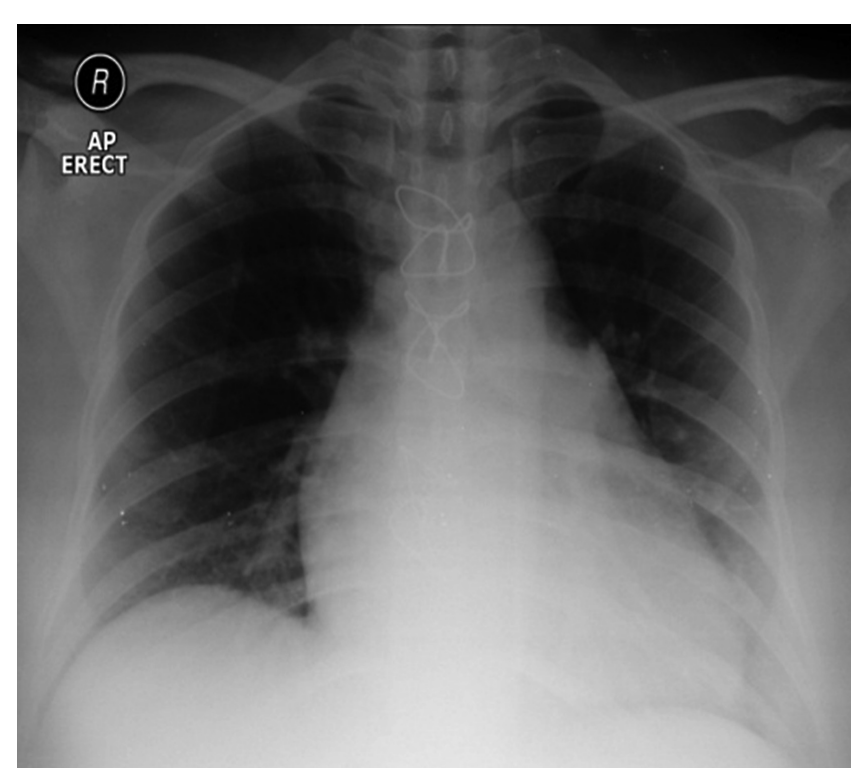

Recurrence of
Intravenous
Leiomyomatosis

223

Figure 1.

Chest X-ray

Preoperative Shows

Cardiothoracic Ratio

Is $55 \%$, There Was

Slight Left Pleural

Effusion, Dilation

Aortic Segment, and

Normal Pulmonal

Segment

valve abnormality except mild tricuspid regurgitation with tricuspid valve gradient (TVG) being $21 \mathrm{mmHg}$.

Venography was done through iliac vein to IVC that resulted in a mobile tumor mass in the IVC mouth which partially flew into the right atrium, and partially blocked the IVC mouth. Tumor size was $6.4 \mathrm{~cm} \times 4.8 \mathrm{~cm}$. Abdominal MSCT resulted in a residual soft tissue mass (leiomyoma) along the IVC extended to the right atrium. The tumor mass size in the IVC and the right atrium was bigger compared. Right major psoas muscle was suspected to be adhered to the IVC wall. Uterine myoma, which was not involved with the IVC, the aorta, or the common iliac artery, was bigger. TEE was also done that resulted in a mass in the right atrium, size $4.8 \times 3.6 \mathrm{~cm}$, which flew from the IVC that caused dynamics obstructed in the tricuspid valve (Figure 2).

Two weeks after admission, the patient underwent the first staged emergency operation collaborated by cardiothoracic surgeon and gynecologist due to total obstruction of tumor mass in superior vena cava (SVC), right atrium, and right ventricle. A median sternotomy was done. The sternum was cut with a sternal saw, and retracted. Adhesion between pericardium and right atrium was identified, and then released. Cardiopulmonary bypass (CPB) was applied between the aorta and the SVC. Next, median laparotomy was done, the IVC was identified, and there was palpabled tumor mass on the intra-IVC and on outerwall of the IVC close to the psoas muscle. Then, the operation was continued by gynecologist performing bilateral oophorectomy. There was no abnormality on uterus. There was adhesion between jejunum and left ovarium. Operation was continued by cardiothoracic surgeon, CPB was started. The right atrium was opened and the tumor mass was clearly visible from the right atrium to the IVC with the size of approximately $6 \mathrm{~cm} \times 5 \mathrm{~cm} \times 6 \mathrm{~cm}$, and then cut off. The right atrium was closed and countinued with the IVC. Exploration was done along the IVC that revealed a tumor the size of $28 \mathrm{~cm} \times 6 \mathrm{~cm}$, and then the tumor mass was excised by making incision on the IVC until the iliac vein. The tumor mass was whitish in color. The tumor originated from the pelvic cavity penetrating the vessels at right the iliac 
Proceedings of MICoMS 2017

\section{4}

\section{Figure 2.}

Venography. Arrow: Mobile Tumor Mass in the IVC Which Partially Flew into Right Atrium, and Partially Blocked the IVC Mouth. Tumor Size was $6.4 \mathrm{~cm} \times$ $4.8 \mathrm{~cm}$ in the Right Atrium

\section{Figure 3.}

(a) Abdominal MSCT resulted residual soft tissue mass

(leiomyoma) along IVC extended to right atrium. Arrow: Mass from IVC extended to RA. (b) TEE $0^{\circ}$ view. Arrow: mass in the right atrium, size $4.8 \times 3.6 \mathrm{~cm}$ which is flew to the RV that caused dynamics obstructed to the tricuspid valve

vein. Venous repair was performed from the IVC until the iliac vein. Haemorrhage was controlled by MRDH patch. Hemodynamic was stable and weaning of CPB was done. Wound was closed level by level (Figure 3).

Intraoperative TEE revealed decreasing movement of the LV due to high dose of inotropes, very poor contractility of the right ventricle, and dilatation of right atrium and ventricle. There was a mild tricuspid regurgitation with TVG being $20 \mathrm{mmHg}$, no pulmonary hypertension, no clot in IVC, and there was kissing left ventricle. Patient was transferred to ICU for recovery and prepared for next-stage operation (Figure 4).
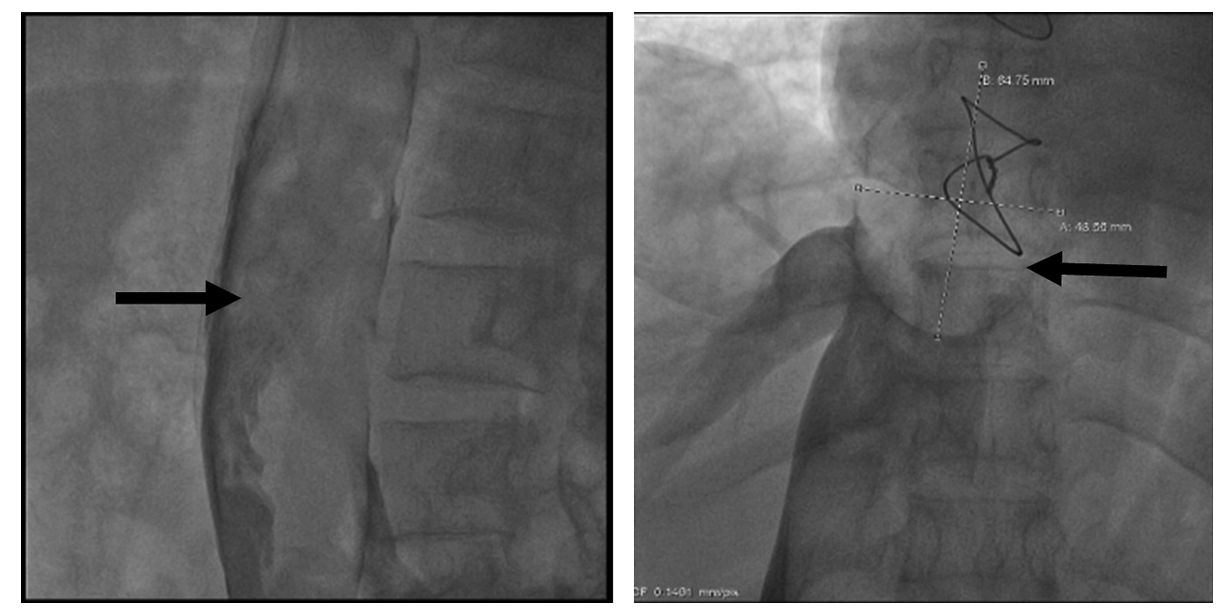

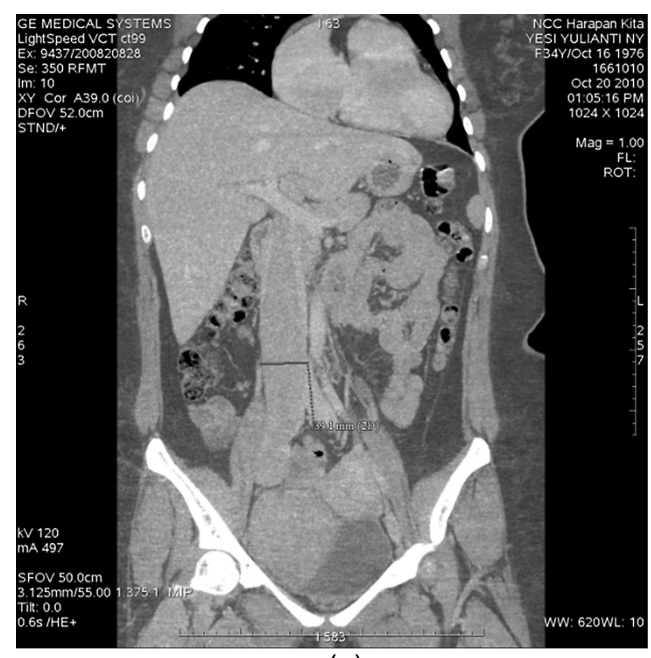

(a)

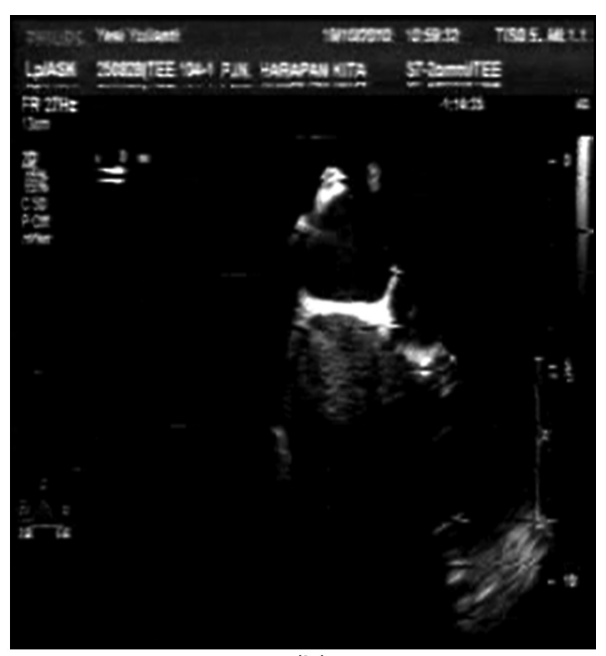

(b) 


\section{Discussion}

Tumors of the heart are uncommon in everyday surgical practice. Primary cardiac tumors are rare and usually benign (Rosenberg et al., 1988). These may require complete resection for diagnostic purposes and to avoid complications, including right heart failure or RV outflow tract obstruction (Galvin et al., 2010).

Leiomyoma are the most common benign tumor of the uterine myometrium. Intravenous benign leiomyoma with extension to the cardiac structures is a rare diagnosis. Recurrence has been notified unless a completed resection, including hysterectomy, is performed. However, even with complete hysterectomy, recurrence may also occur (Filsoufi et al., 2002; Maylar et al., 2009).

Intracardiac localization is seen in approximately $10 \%$. The tumor most commonly enters through the lumen of the iliac vein, and grows into the IVC, sometimes reaching the right atrium, the ventricle, the and pulmonary artery (Topcuoglu et al., 2004; Virzi et al., 2007). The first report of intracardiac extension of this entity was published in 1907 by Durck and Hormann from an autopsy analysis, 113 cases have been published since then (Thukkani et al., 2005). Eleven cases of extension to the level of the heart have been reported and resected, of which only four have complete excisions. At least two of the incomplete excisions returned with recurrence of symptoms and subsequently underwent re-excision (Rosenberg et al., 1988).

As was found in our patient, first time she had uterine leiomyoma extirpation in 2008, and then also had metastasizing leiomyoma to the IVC and undergone extirpation in the same year. As with other suitable cardiac neoplasms, surgical excision was warranted in our patient for therapeutic and diagnostic reasons. We present images of Thorax CT-scan and initially diagnosed as right atrial myxoma. To our knowledge, cardiac metastasis from a benign metastasizing leiomyoma has been reported only once previously (Clement, 1988). And we did not find any other site of metastasizing in our patient.

The exact etiology of this tumor is unknown. Analysis of all the cases reveals that this entity has occurred only in females, and that most are white and premenopausal. Evidence for a hormonal influence has been found. Tierney has suggested that all patients should be maintained on anti-estrogen therapy to minimize recurrence (Rosenberg et al., 1988; Filsoufi et al., 2002; Topcuoglu et al., 2004). In our patient who had primary infertility, and taking of hormonal chemicals as contraception since 8 years, and it can become a predicting factor for getting recurrence of IVL.

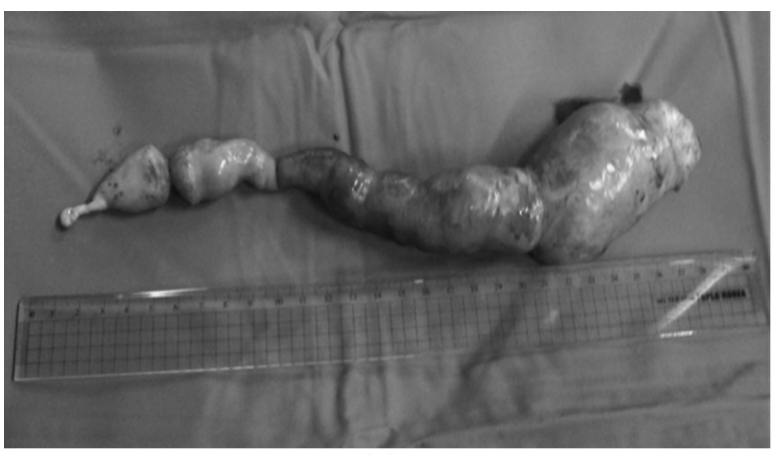

(a)

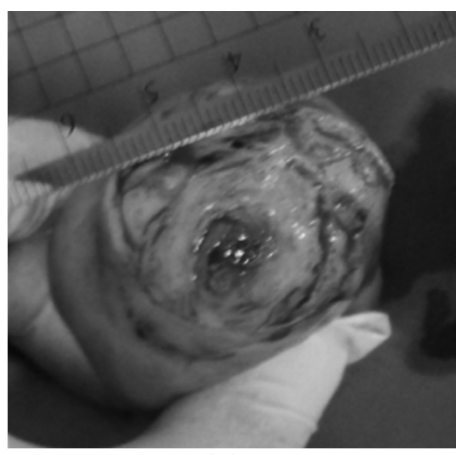

(b)
Recurrence of Intravenous Leiomyomatosis

225

$-$ 
Proceedings of MICoMS 2017

\section{6}

Surgical intervention is generally indicated on the basis of right-sided heart failure or risk of embolism, or both. To treat gynecologic neoplasms extending into the IVC up to the right atrium is well described in the elective setting (Katsumata et al., 1998; Rivera et al., 2004; Sapmaz et al., 2008; Nasu et al., 2009). Single-staged cardiac and abdominal approach by a sterno-laparotomy is usually preferred to obtain radical resection. However, these rare entities may sporadically be present as emergencies and cause sudden hemodynamic instability and shock (Luciani et al., 2009).

In the most recent series and in our experience, the combined single-staged approach under sternal-laparotomy is preferred for the management of caval tumors originating from the uterus, the ovaries, because it allows complete removal of the mass from both the large veins and the abdomen or the pelvis (combined hysterectomy and oophorectomy) for hormonal (Katsumata et al., 1998; Rivera et al., 2004; Sapmaz et al., 2008; Luciani et al., 2009; Maylar et al., 2009; Galvin et al., 2010). Our strategy to this patient was the same as the literature mentioned earlier.

In the patient we have described, the hemodynamic instability prompted treatment solely of the intracardiac portion of the tumor. Additional determinants of such strategy were the incomplete data on the proximal origin of the tumor and the compromised general condition of the patient, who had scarce possibilities to tolerate a prolonged stern-laparotomy procedure. Elective management in our previous experience of patients with intracardiac uterine leiomyomatosis extending to the right atrium through the IVC included a singlestaged operation involving two surgical teams and complete resection of the tumor. The postoperative course was uneventful.

Various surgical approaches have been used, including a one-stage radical resection or a two-stage resection. One-stage resections have been reported on bypass with normothermia or with hypothermic circulatory arrest. Two-stage procedures have been advocated for extensive leiomyomatosis (Clement, 1988; Filsoufi et al., 2002; Topcuoglu et al., 2004; Thukkani et al., 2005; Galvin et al., 2010). Surgical strategy with norm thermic CPB, as in the present patient, has shown that this approach guarantees several advantages, including (1) the possibility to perform extensive cavotomy if required, (2) the ability to evaluate the sub-hepatic veins for possible involvement, and (3) a bloodless operative field. Ongoing $\mathrm{CPB}$ during the entire procedure should probably be reserved for rare instances of limited intravenous caval thrombosis or tumor without extension into the lower portions of the vena cava (Clement, 1988; Thukkani et al., 2005; Sapmaz et al., 2008).

Intraoperative TEE should always be performed in these patients before and after CPB. A second operation was then needed to obtain radical excision (Kocica et al., 2005; Sapmaz et al., 2008; Nasu et al., 2009).

In our patient we did two-staged resection, first we did resection of the tumor in the Robby using CPB and no potential to injured seen at the tricuspid valve leaflets, and then was continued with laparotomy, resect the IVC to remove the tumor and gynaecologic operations to expose the patient to a lower postoperative bleeding risk. The correct diagnosis was made during the surgery, therefore a two-staged resection was planned. Surgical resection is the best treatment for intracardiac extension of intravenous leiomyoma. We recommend iliac venotomy to remove the ilio-caval portion of the tumor in both stages of operations and oophorectomy. Intraoperative TEE revealed poor LV and RV contractility, mild tricuspid regurgitation with TVG being $20 \mathrm{mmHg}$ and kissing LV.

Because a complete removal of the tumor is mandatory, an adequate preoperative diagnosis should provide detailed information about the tumor localization, extravascular and intravascular diameters, site of vascular entry, and patency of iliac and femoral veins. The optimum and safest strategy is a single or two-stage procedure with an abdominal 
approach and subsequent thoracic approach. The abdominal stage should ensure complete extravascular tumor excision and control of the vascular entry site. The thoracic stage, preferably with short total $\mathrm{CPB}$ on the beating heart, should allow easy tumor withdrawal from the right cardiac chambers and the IVC (Roques et al., 2001; Kocica et al., 2005).

\section{Conclusion}

Correct diagnosis at the first presentation is therefore crucial to plan the treatment strategy under elective conditions. A high degree of suspicion should be probably kept when, in the presence of an intravenous inferior caval mass, historical elements and CT imaging features do not corroborate the hypothesis of caval thrombosis.

Preoperative studies must be performed and should include at least an inferior vena cavogram. Complete excision is necessary to avoid recurrence. The use of hormonal manipulation to modify the intra-abdominal portion is unknown but should be considered after partial resection. The correct diagnosis was made during the surgery, therefore a twostage resection was planned. Surgical resection is the best treatment for intracardiac extension of intravenous leiomyoma. We recommend iliac venotomy to remove the ilio-caval portion of the tumor in both stages of operations.

\section{References}

Clement, P.B. (1988). "Intravenous Leiomyomatosis of the Uterus". Pathology Annual, Vol. 23, Pt. 2, pp. 153-183.

Filsoufi, F., Farivar, R.S., Anderson, C., Santerre, D. and Adams, D.H. (2002). "Renal Vein Injury Complicating Removal of Intravenous Leiomyoma”. Journal of Thoracic and Cardiovascular Surgery, Vol. 123, No. 4, pp. 820-822.

Galvin, S.D., Wademan, B., Chu, J. and Bunton, R.W. (2010). "Benign Metastasizing Leiomyoma: A Rare Metastatic Lesion in the Right Ventricle". Annals of Thoracic Surgery, Vol. 89, No. 1, pp. $279-281$.

Katsumata, T., Shinfeld, A., Houel, R. and Westaby, S. (1998). "Pelvic Leiomyoma in the Right Atrium", Annals of Thoracic Surgery, Vol. 66, No. 6, pp. 2095-2096.

Kocica, M.J., Vranes, M.R., Kostic, D., Kovacevic-Kostic, N., Lackovic, V., Bozic-Mihajlovic, V., Velinovic, M.M., et al. (2005). "Intravenous Leiomyomatosis with Extension to the Heart: Rare or Underestimated?", Journal of Thoracic and Cardiovascular Surgery, Vol. 130, No. 6, pp. 17241726.

Luciani, N., Anselmi, A., Glieca, F., Martinelli, L. and Possati, G. (2009). "Diagnostic and Surgical Issues in Emergency Presentation of a Pelvic Leiomyoma in the Right Heart". Annals of Thoracic Surgery, Vol. 87, No. 5, pp. 1589-1592.

Maylar, N.M., Piotrowski, A.J., Erbel, R. and Schmitz, K.J. (2009). “Giant Right Atrial Mass: Intracardiac Extension of Intravenous Leiomyoma 18 Years After Hysterectomy". Annals of Thoracic Surgery, Vol. 88, No. 2, p. 684.

Nasu, K., Tsuno, A., Takai, N. and Narahara, H. (2009). "A Case of Benign Metastasizing Leiomyoma Treated by Surgical Castration Followed by an Aromatase Inhibitor, Anastrozole". Archives of Gynecology and Obstetrics, Vol. 279, No. 2, pp. 255-257.

Rivera, J.A., Christopoulos, S., Small, D. and Trifiro, M. (2004). "Hormonal Manipulation of Benign Metastasizing Leiomyomas: Report of Two Cases and Review of the Literature". Journal of Clinical Endocrinology and Metabolism, Vol. 89, No. 7, pp. 3183-3188.

Roques, F., Sanchez, B., Bucher, B. and Larivière, J. (2001). "Role of Pre-Operative Assessment in the Surgical Management of Leiomyoma Extended to the Right Heart Chambers: A Compendium of Information From Isolated Reports", European Journal of Cardio-Thoracic Surgery, Vol. 19, No. 4, pp. 522-524. 
Proceedings of Rosenberg, J.M., Marvasti, M.A., Obeid, A., Johnson, L.W. and Bonaventura, M. (1988). "Intravenous MICoMS 2017 Leiomyomatosis: A Rare Cause of Right Sided Cardiac Obstruction”. European Journal of Cardio-Thoracic Surgery, Vol. 2, No. 1, pp. 58-60.

Sapmaz, F., Ergin, M., Katrancioglu, O., Gonlugur, T., Gonlugur, U. and Elagoz, S. (2008). "Benign Metastasizing Leiomyoma”, Lung, Vol. 186, No. 4, pp. 271-273.

Thukkani, N., Ravichandran, P.S., Das, A. and Slater, M.S. (2005). "Leiomyomatosis Metastatic to the Tricuspid Valve Complicated by Pelvic Hemorrhage”. Annals of Thoracic Surgery, Vol. 79, No. 2, pp. 707-709.

Topcuoglu, M.S., Yaliniz, H., Poyrazoglu, H., Tokcan, A., Demir, S.C., Bozkurt, A. and Zeren, H. (2004). "Intravenous Leiomyomatosis Extending into the Right Ventricle after Subtotal Hysterectomy". Annals of Thoracic Surgery, Vol. 78, No. 1, pp. 330-332.

Virzì, G., Ragazzi, S., Bussichella, F., D’Agati, P., Caputo, S., Scaravilli, F. and Piazza, D. (2007). "Intravenous Leiomyomatosis Extending from the Inferior Caval Vein to the Pulmonary Artery”. Journal of Thoracic and Cardiovascular Surgery, Vol. 133, No. 3, pp. 831-832.

\section{Corresponding author}

Yopie Afriandi Habibie can be contacted at yopie98@yahoo.com 